\title{
On the Effectiveness of Switched Beam Antennas in Indoor Environments
}

\author{
Marc Blanco $^{2}$, Ravi Kokku ${ }^{1}$, Kishore Ramachandran ${ }^{3}$, Sampath Rangarajan ${ }^{1}$, \\ and Karthik Sundaresan ${ }^{1}$ \\ 1 NEC Laboratories America, Princeton, NJ \\ 2 Rice University, Houston, TX \\ 3 Rutgers University, New Brunswick, NJ
}

\begin{abstract}
Switched beam antennas are an attractive extension to indoor wireless LANs due to their increased signal gain in a chosen direction; the gain can be exploited for improving wireless link quality, node localization and increasing spatial reuse. However, indoor environments are susceptible to multipath reflections that may reduce the degree of directionality of the antennas. To this end, in this paper, we address the following questions that have not been explored well in the open literature: how directional in reality is a beam with a switched beam antenna in a reflection-rich environment, and what are the implications of the observed directionality on spatial reuse and node localization? And how does the directionality get affected with the characteristics of a beam such as main and side lobe width, and front to side lobe ratio? We present results of measurements in a real office setting with a switched beam antenna built out of an 8-element phase array.
\end{abstract}

\section{Introduction}

Several research works demonstrate the benefits of directional antennas in wireless networks, especially for better link quality and spatial reuse $[6,9,11-13,15$, 16], localization [10,14] and security [4]. All these works exploit the ability of a directional antenna to focus the transmission energy in a particular direction and suppress the energy in unwanted directions; the ability is often loosely termed as the directionality of the antenna. Most of these works assume environments such as outdoors where the antenna provides close to the desired directionality, and presented analytical, simulation and a few prototype $[9,12,13]$ studies to demonstrate the benefits. In the context of indoor environments, conventional wisdom appears to be that the benefits of directional antennas may not be as dramatic as outdoors due to multipath reflections.

Two recent trends, however, motivate renewed interest in using directional antennas in indoor environments. First, with the recent popularity of enterprise WLANs for diverse applications such as VOIP, mainstream office applications, video conferencing and streaming, interest is increasing in the industry for tapping the benefits of directional antennas in indoor enterprise WLANs (e.g. See 
Ruckus Wireless [3]). Second, the technology for achieving directionality with antennas is becoming cheaper and readily available to make it attractive for incorporating into WLAN products easily $[1,3]$. Depending on the features and flexibility provided, directional antennas can be classified into patch and sectorized antennas, switched beam antennas, and adaptive beamforming antennas. While patch and sectorized antennas are designed to focus the antenna beam pattern in one fixed direction, switched beam antennas provide several fixed beams out of which one is chosen for transmission and reception, and adaptive beam antennas adapt beams dynamically in signal space to minimize interference to as many other nodes in the network as possible.

In this paper, we focus on studying switched beam antennas in indoor environments. They provide a good tradeoff among the available antenna technologies; they are less bulky than a collection of patch antennas for providing the same amount of network coverage, and they are simpler to implement and incorporate than adaptive beamforming antennas that require significant channel feedback from receivers for forming appropriate beams dynamically at a transmitter. With respect to switched-beam antennas, this paper answers through measurements in a realistic setting a set of basic and important questions: How directional in reality is a beam with a switched beam antenna in a reflection-rich environment? How does the directionality get affected with the characteristics of a beam such as main and side lobe width, front to side lobe ratio, and location of clients? The only research effort that we are familiar of in the indoor context is [5] that employs $10^{\circ}$ beams and focuses on improving link quality; it does not address the more generic questions we ask.

The ability of switched-beam antennas to form directional beams that suppress energy in several directions contributes to increased simultaneous transmissions in the network, often referred to as spatial reuse. Further, the increased signal strength in the main beam direction towards the clients, helps localize the client within the angular width of a beam. We study the degree of directionality offered by switched-beam antennas in the context of spatial reuse and localization. In particular, we make the following key observations. (1) The notion of directionality is different for different applications such as spatial reuse and localization, and hence the traditional approach of using "gain over an omnidirectional antenna" to quantify directionality [13] is not comprehensive. (2) Although reflections in indoor environments increase the interference in more directions than ideal, there can be several locations in an indoor environment where a directional beam indeed suppresses interference, thereby making spatial reuse possible. (3) For localization, while most of the clients get localized correctly, a few clients get wrongly localized mainly because of a small difference in RSSI (Received signal strength) between the best beam for a client and the beam in the actual direction of the client., thereby necessitating intelligent beam resolution mechanisms. (4) Finally, while our experiments verify that thinner beamwidths do yield greater directionality, they do not completely eliminate the impact of indoor reflections, thereby reinforcing the importance of the above implications in indoor environments even with thin beams. 


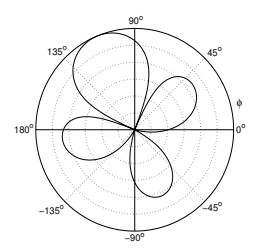

(a) Pattern 1

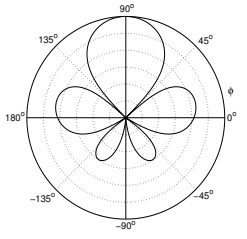

(b) Pattern 2

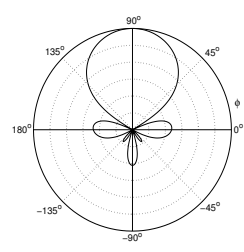

(c) Pattern 3

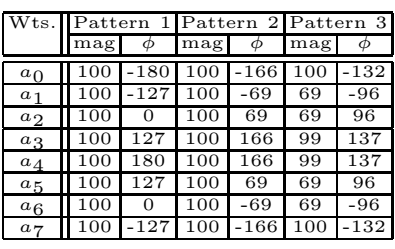

(d)

Fig. 1. Antenna beam patterns (a, b, c) and the corresponding element weights (d)

The rest of the paper is organized as follows. Section 2 describes the basics of switched beam antennas. Section 3 presents the experimental methodology that we use to study the directionality of a switched beam antenna in a typical indoor environment. Section 4 presents results and our interpretations from our experiments. Section 5 summarizes the paper.

\section{Background}

A common way of realizing switched beam or adaptive beamforming antennas is by using phase array antennas. Phase array antennas consist of an array of antenna elements, the signals sent to which are weighted in both magnitude and phase. The combination of these weighted signals when radiated by the elements simultaneously form the antenna radiation pattern that can often be of complex shapes depending on the weights. In general, the antenna radiation pattern for an $N$-element array is represented by,

$$
A(k)=a_{0} \exp ^{j k d_{0}}+a_{1} \exp ^{j k d_{1}} \ldots+a_{N-1} \exp ^{j k d_{N-1}}
$$

where $a_{n}$ is a complex quantity corresponding to the magnitude and phase of the weight applied to the $n^{\text {th }}$ antenna element, $\mathrm{k}=2 \pi$ and $d_{n}$ represents the displacement of the element from a point of reference respectively. The applied weights help reinforce energy in a particular direction, thereby producing a high SNR (Signal to Noise Ratio) over an omni pattern in the desired direction contributing to a direction/array gain. Since phase array antennas available in practice cannot completely eliminate the energy radiated in undesired directions, they do result in some spill-over of energy in the unwanted directions, which are referred to as the side-lobes. As the main lobe is made more thin (focused), the array gain increases. However, it also increases the spill-over into side lobes. This trade-off is captured in the form of front-side lobe ratio of any directional antenna.

To realize a switched beam antenna, several such beam patterns can be generated with a phase array antenna such that they cover the entire azimuth $\left(360^{\circ}\right)$, and a specific beam pattern is dynamically chosen from the available set during operation. In this paper, we use Fidelity Comtech's Phocus Array [2] for our experiments. This antenna is a circular array of eight elements arranged in a regular octagon. The antenna is electronically steerable, i.e., a specific beam 
pattern out of the several precomputed beams can be chosen from software on the fly. Figure 1(a),(b, (c) show different patterns created with the Phocus array antenna, and the corresponding weights are shown in Figure 1(d). Patterns (a) and (b) - provided with the phase array by Fidelity Comtech - have a half-power beamwidth of $45^{\circ}$ and a front to side lobe ratio of $8 \mathrm{~dB}$. Pattern (c), that we generated, has a half-power beamwidth of $60^{\circ}$ and a higher front to side lobe ratio of $18 \mathrm{~dB}$. Note that with an $\mathrm{N}$ element antenna, the minimum main lobe width we can achieve is approximately $360^{\circ} / \mathrm{N}$. Hence, for thinner beamwidths than $45^{\circ}$, we need greater than eight elements in the antenna.

\section{Experimental Methodology}

In this section, we describe our testbed and the methodology for studying the effectiveness of switched-beam antennas in improving spatial reuse and node localization. Our experiments evaluate the effects of different parameters such as beamwidth, front-to-side lobe ratio, node locations (line-of-sight or non-lineof-sight) and transmit power on the directionality of switched beam antennas.

Metric The ideal beam pattern for a switched beam antenna is a single strong beam producing a high SNR (over omni pattern) in the direction of the receiver with no or negligible side-lobes in all the other directions. However, practical beam patterns do have considerable side-lobes. Further, multipath propagation indoors complicates the situation by resulting in reflected components of the main beam and the side-lobes. Thus, the three main components contributing to the directionality of a switched beam antenna for a given receiver are (a) "very few" beams with a large received signal strength $S_{S}$ over the omni signal strength $S_{O}\left(S_{S}>S_{O}\right)$, (b) "large" number of beams with a large reduction in received signal strength compared to omni $\left(S_{S}<S_{O}\right)$, since this represents interference suppression in several directions, and (iii) the beam with the largest gain $\left(S_{S}>\right.$ $S_{O}$ ) coinciding with the geographic beam oriented towards the receiver.

Though there are multiple components to directionality, not all components may be required by applications and the specific components impacting applications varies with the nature of the application. For improving spatial reuse, we require the first two components of directionality to be satisfied, while it is not important the the strongest beam coincide with the main geographic beam. On the other hand, for a localization application, wherein it may be acceptable if the signal spills over in several beam directions, it is imperative that the third component be satisfied.

Setup Our experimental setup is shown in Figure 2. The setup contains one AP connected to the Phocus array antenna, and 11 receiver nodes distributed in our office building in different office rooms and cubicles. Each receiver node is a small form-factor PC equipped with mini-PCI $802.11 \mathrm{a} / \mathrm{b} / \mathrm{g}$ cards based on the Atheros 5212 chipset. The nodes run Linux kernel v2.4.26 and the MadWiFi driver [8] and their WLAN Radios connect to external OMNI antennas with a gain of $6 \mathrm{dBi}$. 


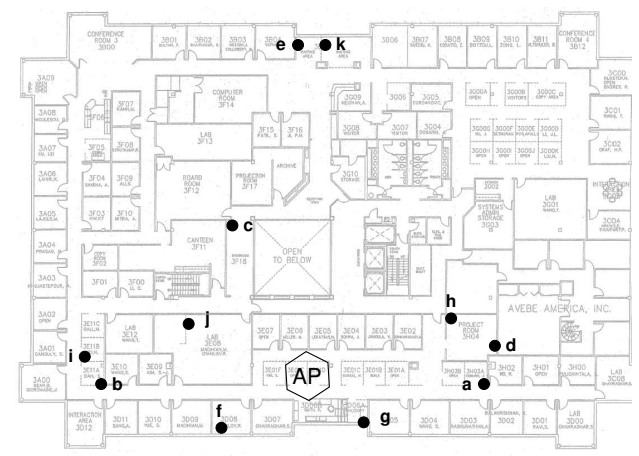

Fig. 2. Testbed Setup. Black dots indicate the locations of receiver nodes.

For the Phocus array antenna, we use three beam pattern sets each containing eight patterns for eight directions to cover the entire circle (geographical area) around the AP. The patterns in each set are shifted by $45^{\circ}$ from one another such that there is atleast one pattern that geographically covers each receiver. Pattern set 1 contains patterns like Figure 1(a), set 2 like Figure 1(b), and set 3 like Figure 1(c). We also generate an omni-directional pattern for comparison. All experiments are done on channel 6 in the $2.4 \mathrm{Ghz}$ spectrum at night to avoid disturbing and getting disturbed by regular office usage of the channel.

In all the experiments, the AP sends 128 byte UDP broadcast packets using the Click router package [7], and the receivers execute tcpdump in monitor mode. The AP utilizes the 802.11 pseudo-IBSS (Independent Basic Service Set) mode, in conjunction with monitor mode, which allows (a) all nodes to communicate directly and (b) the transmission of 802.11 broadcast frames at specified bitrates from user-space. The AP chooses different directions in turn and transmits, and the receivers act as sensors by collecting data that helps us determine the directionality of the antenna.

\section{Evaluation}

In this section, we present several results that demonstrate in indoor environments (1) the degree of directionality obtained with a switched beam antenna, (2) the potential for spatial reuse, and (3) the accuracy of node localization.

Directionality We first perform experiments to study the degree of directionality provided by the phase array antenna. In this set of experiments, the AP uses one of the three sets of beam patterns described in Section 3. For each set of beam patterns, the AP chooses a pattern in turn and broadcasts 1000 packets of size 128 bytes at $2 \mathrm{Mbps}$ bitrate. From the received packets for each beam, we calculate the average RSSI on each receiver when the AP chooses the beam. We also repeat the experiment with an omni-directional pattern (OMNI). We 


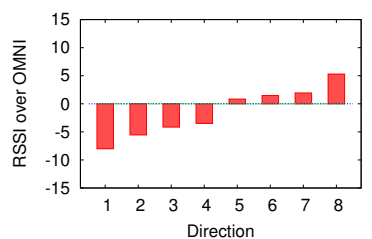

(a) Location a, LOS

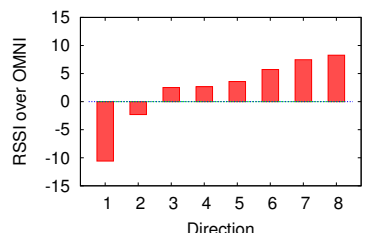

(d) Location d, NLOS

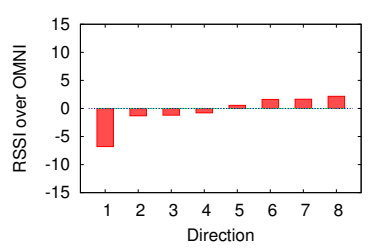

(g) Location g, LOS

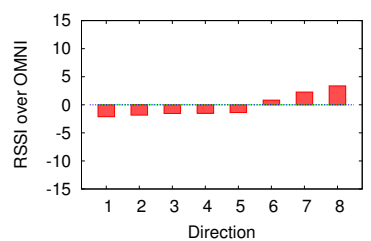

(b) Location b, NLOS

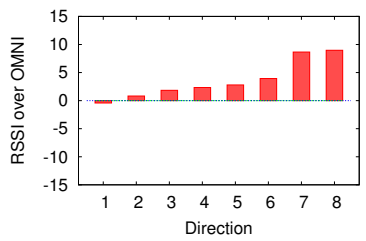

(e) Location e, NLOS

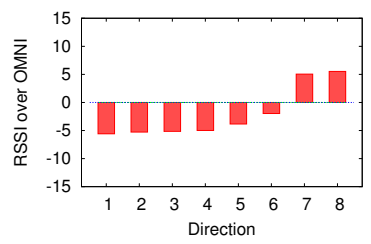

(h) Location h, LOS

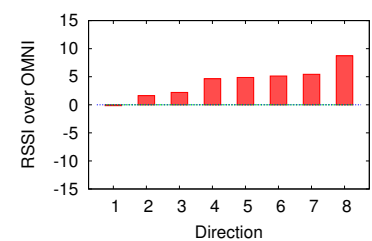

(c) Location c, NLOS

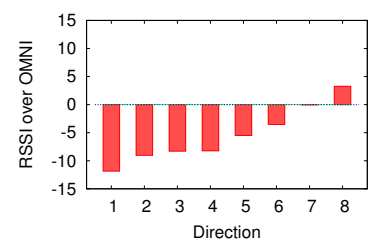

(f) Location f, LOS

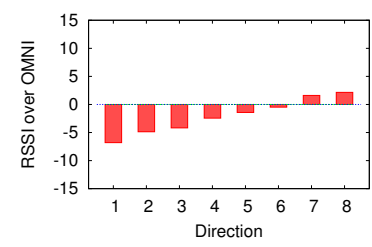

(i) Location i, NLOS

Fig. 3. RSSI over OMNI (in dB) at each receiver with different beams (directions).

then calculate the difference in RSSI between each beam and OMNI; if the difference is lower than 0 for beam $j$ for a receiver, it means that the AP causes less interference on the receiver when it uses the beam $j$ instead of OMNI.

Figure 3 shows our observations on different receivers for pattern set 3 . We include the graphs for pattern sets 1 and 2 in the appendix. As conventional wisdom says, we indeed find that there are several directions in which there is significant interference compared to OMNI 3(c,d,e). This observation is true even with beamwidths as low as $45^{\circ}$ (with pattern sets 1 and 2) and front-side lobe ratio as high as $18 \mathrm{~dB}$ (with set 3 ). Also, in a few cases, the strongest beam does not correspond to the main geographic beam 3(b,e,g). However, there are also many cases $(\mathrm{a}, \mathrm{b}, \mathrm{f}, \mathrm{g}, \mathrm{h}, \mathrm{i})$, and notably $(\mathrm{a}, \mathrm{f}, \mathrm{h})$, where RSSI is significantly lower than OMNI in several directions. In a large number of cases, the strongest beam coincides with the geographic beam $3(\mathrm{a}, \mathrm{c}, \mathrm{d}, \mathrm{f}, \mathrm{h}, \mathrm{i})$. With respect to the first two components of directionality, we find that the clients that indicate good directionality $3(\mathrm{a}, \mathrm{f}, \mathrm{h})$ are those that have a strong line-of-sight (LOS) component. However, this does not necessarily mean that all clients with a LOS component will show good directionality (e.g.3(g)) since it is possible for the multipath reflections to weaken the LOS component. With respect to clients with nonline-of-sight (NLOS) components, we find that they suffer in directionality with 


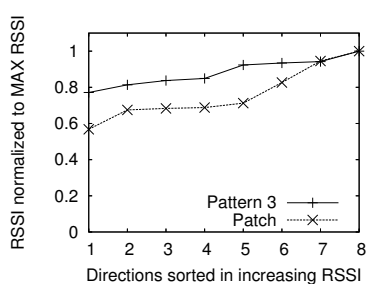

(a) Location a

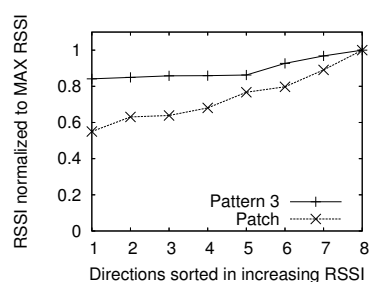

(b) Location b

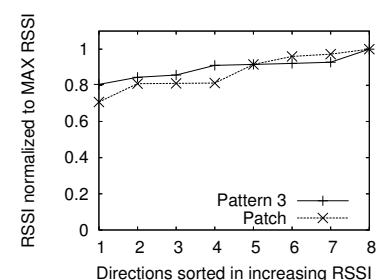

(c) Location c

Fig. 4. Directionality with a $17^{\circ}$ patch antenna.

respect to the first two components. When it comes to strongest beam coinciding with the geographic beam, we find that this component of directionality is not dependent on the presence of a strong LOS component, with both LOS and NLOS clients showing good directionality $3(\mathrm{a}, \mathrm{c}, \mathrm{d}, \mathrm{f}, \mathrm{h}, \mathrm{i})$.

These observations indicate that the presence of multipath in indoor environments does not completely negate the directional benefits of switched beam antennas. Further, the availability of a strong LOS component does not indicate better directionality and vice versa. In fact, depending on the specific application considered and the specific components of directionality required by the application, both LOS and NLOS clients can potentially exploit directionality. However, this would also require that the solutions designed for these applications take into account the implications of multipath reflections. To understand the importance of these implications, we further conducted experiments comparing the directionality of the $60^{\circ}$ pattern produced by the phased arrays with that of a $17^{\circ}$ pattern produced by a much bulkier patch antenna. We use a patch antenna here since an 8-element phase array antenna that we have can only generate a minimum beamwidth of $45^{\circ}$. The results presented in Figure 4 measure the normalized RSSI (sorted and normalized to maximum) as a function of different directions for three different clients (a), (b) and (c). The graphs demonstrate that thinner beam-widths do provide better directionality (by showing that the patch line is lower on the left than pattern 3 ).

Spatial Reuse Ideally, determining the exact amount of spatial reuse possible is hard since it depends on several factors such as network topology, propagation characteristics in the indoor environment, MAC implementation, etc. Hence, we take an indirect approach to argue the potential for spatial reuse with switchedbeam antennas. We observe that for spatial reuse, it is not just sufficient for the RSSI to be lower than OMNI, but should be lower by a considerable amount. Clearly, cases such as in Figure 3(a,f,h,i) show significant reduction in RSSI in multiple directions, thereby making spatial reuse possible. Just for illustration, we plot in Figure 5, the number of directions that are lower than OMNI by more than $3 \mathrm{~dB}$ (every $3 \mathrm{~dB}$ decrease represents halving the power, and hence interference). The graph shows that five locations have at least three directions (about 135 degrees) where interference is lower than OMNI by more than $3 \mathrm{~dB}$, thereby indicating chances of spatial reuse. 


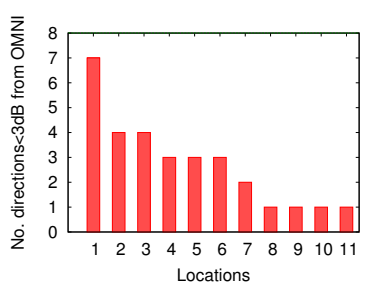

(a) Pattern 1

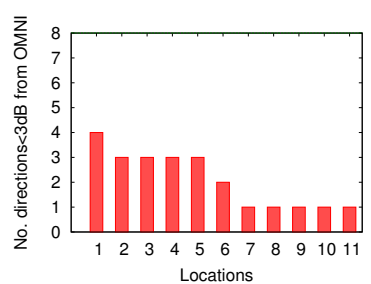

(b) Pattern 2

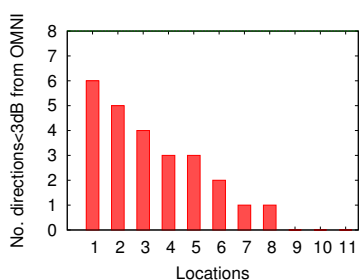

(c) Pattern 3

Fig. 5. Potential for Spatial Reuse with Switched-beam antennas.

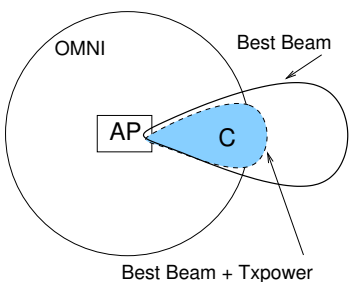

(a)

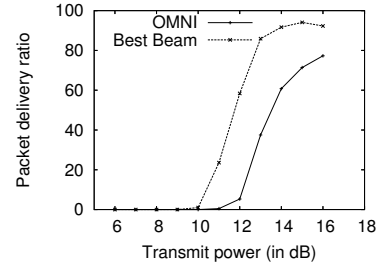

(b) Location b

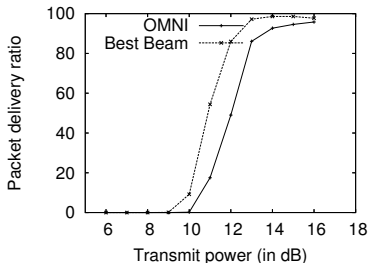

(c) Location $\mathrm{h}$

Fig. 6. Combining directionality and transmit power control for increased spatial reuse.

Notice, however, that a directional antenna merely changes the area of interference compared to OMNI. To illustrate, Figure 6(a) shows a simplified picture of the area of interference with OMNI and directional beam patterns for an (AP,client) transmission. While the pattern "Best Beam" avoided interference in some regions that OMNI doesn't, "Best Beam" also causes interference in regions where OMNI doesn't due to the gain obtained by refocusing the transmission energy. Nevertheless, the AP can reduce the transmit power to the client for achieving the same performance as OMNI and meet the client's requirements. The effective beam looks like the shaded area in the figure that reduces the interference. Note that power control also reduces side lobes that cause interference to other transmissions. Figure 6(b) and (c) support the above proposal for combining transmit power control and directional transmission. In these graphs, we plot for two non-line-of-sight clients the MAC level packet delivery ratio obtained with changing transmit power, when using 54Mbps bitrate with OMNI and the best directional beam. The graphs clearly show that for the same packet delivery ratio, directional beam enables reducing transmit power over OMNI, thereby promoting higher spatial reuse.

Localization Finally, we perform experiments to determine the effectiveness of the antenna in node localization. Most localization techniques assume that a receiver node is in the direction of the beam that produces the highest RSSI at the receiver, which might not be valid in reflection-rich environments. In this experiment, we measure the angle of deviation between the actual direction and the direction with highest RSSI for each of the receivers when the AP transmits 


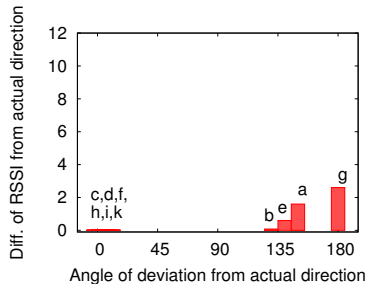

(a) Pattern 1

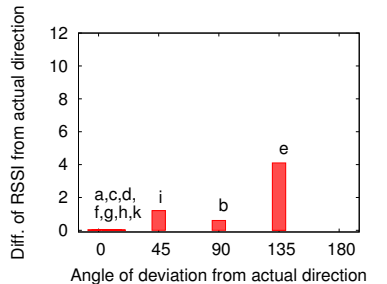

(b) Pattern 2

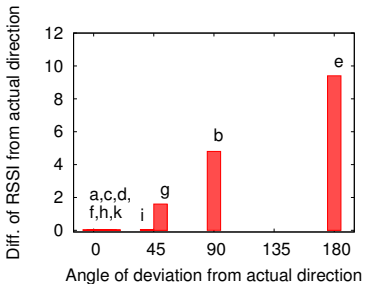

(c) Pattern 3

Fig. 7. Efficacy of localization

with different beams from each pattern set. We also measure the difference in RSSI between the best beam and the beam that points to the actual direction. Figure 7 shows the angle of deviation for each client and the corresponding difference in RSSI (in dB). The graph shows that the patterns position significant number of clients correctly, satisfying the third component of directionality. In fact, some of these clients do not exhibit good interference suppression, but this is not required for localization. A few of the clients get positioned wrongly because of small difference in RSSI. Also, some clients that get wrongly positioned by one pattern get correctly positioned by another pattern. This observation suggests that in reflection-rich environments, just using the beam with highest RSSI may not be the right approach; a localization technique should choose from one of several best beams and even across different pattern sets for better accuracy. Developing such techniques is outside the scope of this paper.

Comparison with MIMO Multiple-input multiple-ouput (MIMO) antenna technology is a popular alternative for indoor environments due to their ability to positively leverage multipath. However, for the specific applications of spatial reuse and node localization that we are interested in, MIMO antennas may still not serve the purpose. This is because (i) in the open-loop mode (no channel state information from receivers) MIMO can only help improve the link performance but cannot contribute to spatial reuse or address node localization, and (ii) in the closed-loop mode (having channel state information from receivers) they require significant feedback overhead and client modifications to enable adaptive beamforming that can address multipath. Nevertheless, a quantitative comparison to MIMO in terms of spatial reuse is an interesting topic for future work that we intend to take up as soon as such hardware and software become friendly for experimentation.

\section{Conclusion}

We study the directionality of a switched beam antenna in indoor environments in the context of spatial reuse and node localization. We make the following key observations. (1) Although reflections in indoor environments increase the interference in more directions than ideal, there can be several locations in an indoor 
environment where a directional beam indeed suppresses interference, thereby making spatial reuse possible. (2) For localization, while several clients get localized correctly, a few clients get wrongly positioned mainly because of a small difference in RSSI between the best beam and the beam in the physical direction of a client, thereby necessitating intelligent beam resolution mechanisms. (3) Finally, while thinner beamwidths do yield greater directionality, they do not completely eliminate the impact of reflections, which reinforces the importance of the above implications in indoor environments even with thin beams.

\section{References}

1. Hyperlink technologies. http://www.hyperlinktech.com.

2. Phocus Array Antenna by Fidelity Comtech. http://www.fidelity-comtech.com/.

3. Ruckus wireless smart antenna. http://www.ruckuswireless.com/.

4. J. M. Carey and D. Grunwald. Enhancing wlan security with smart antennas: a physical layer response to information assurance. In VTC, Sep. 2004.

5. E. Casas, M. Tong Chia da Silva, H. Yin, and Y.-S. Choi. Beam diversity for indoor wlan systems. In VTC 2003.

6. R. R. Choudhury, X. Yang, R. Ramanathan, and N. H. Vaidya. On designing mac protocols for wireless networks using directional antennas. IEEE Trans. Mobile Comput., 5(5):477-491, May 2006.

7. E. Kohler, R. Morris, B. Chen, J. Jannotti, and M. F. Kaashoek. The click modular router. ACM Trans. Comput. Syst., 18(3):263-297, 2000.

8. MADWiFi. Multiband Atheros Driver for WiFi. http://madwifi.org.

9. V. Navda, A. P. Subramanian, K. Dhanasekaran, A. Timm-Giel, and S. Das. Mobisteer: using steerable beam directional antenna for vehicular network access. In MobiSys, 2007.

10. D. Niculescu and R. Badrinath. VOR base stations for indoor 802.11 positioning. In Proc of MobiCom '04, Philadelphia, PA, Sep. 2004.

11. J.-S. Park, A. Nandan, M. Gerla, and H. Lee. Space-mac: enabling spatial reuse using mimo channel-aware mac. In ICC, May 2005.

12. B. Raman and K. Chebrolu. Design and evaluation of a new mac protocol for long-distance 802.11 mesh networks. In MobiCom, 2005.

13. R. Ramanathan, J. Redi, C. Santivanez, D. Wiggins, and S. Polit. Ad hoc networking with directional antennas: a complete solution. IEEE J. Sel. Areas Commun., 23(3):496-596, Mar. 2005.

14. K. Sayrafian-Pour and D. Kaspar. Source-assisted direction estimation inside buildings. In INFOCOM, 2006.

15. R. Vilzmann, C. Bettstetter, D. Medina, and C. Hartmann. Hop distances and flooding in wireless multihop networks with randomized beamforming. In Proc of MSWiM '05, Montreal, Quebec, Canada, Oct. 2005.

16. C. Zhu, T. Nadeem, and J. Agre. Enhancing 802.11 wireless networks with directional antenna and multiple receivers. In Proc. of MILCOM, 2006.

\section{Appendix}




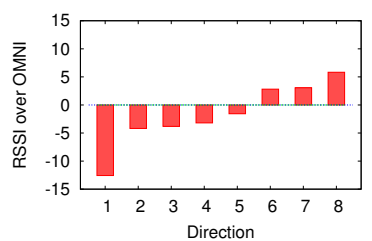

(a) Location a, LOS

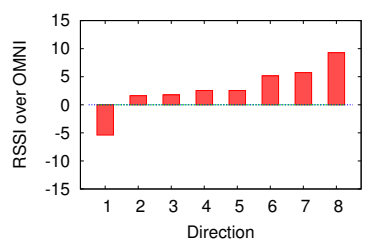

(d) Location d, NLOS

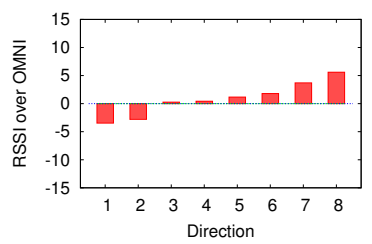

(g) Location g, LOS

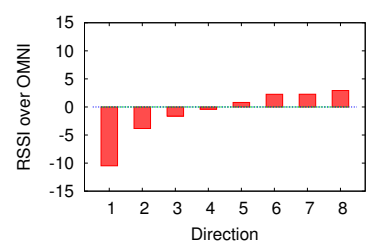

(b) Location b, NLOS

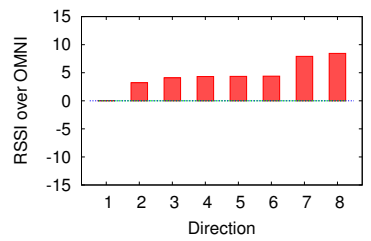

(e) Location e, NLOS

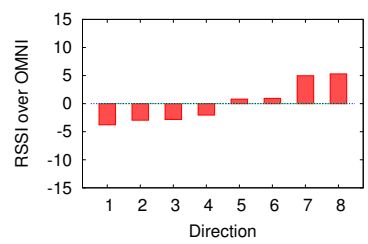

(h) Location h, LOS

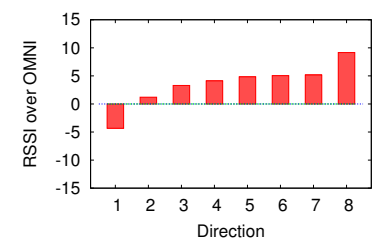

(c) Location c, NLOS

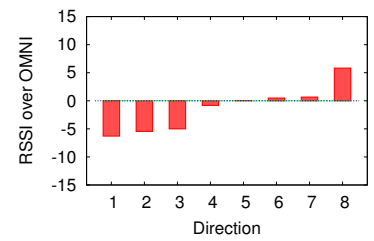

(f) Location f, LOS

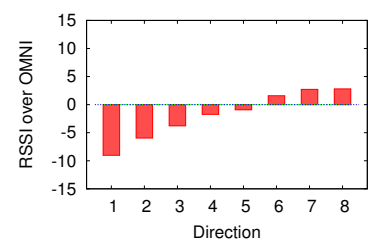

(i) Location i, NLOS

Fig. 8. RSSI over OMNI (in $\mathrm{dB}$ ) at each receiver for pattern set 1 


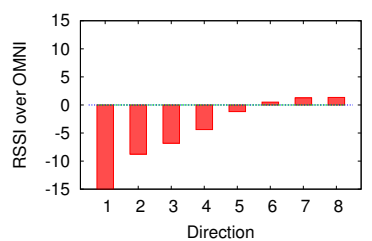

(a) Location a, LOS

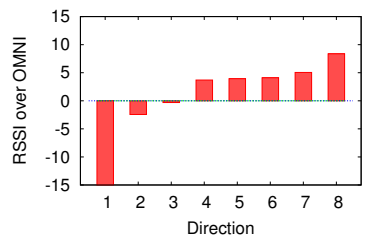

(d) Location d, NLOS

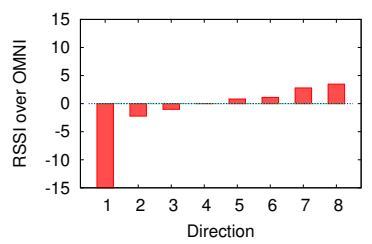

(g) Location g, LOS

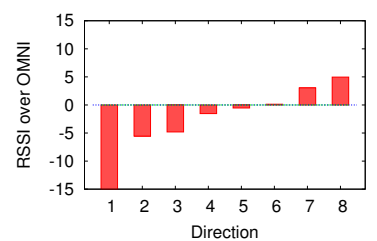

(b) Location b, NLOS

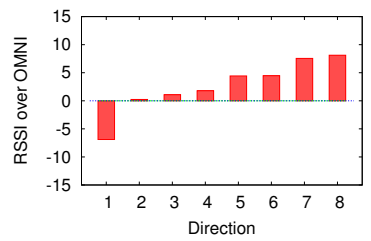

(e) Location e, NLOS

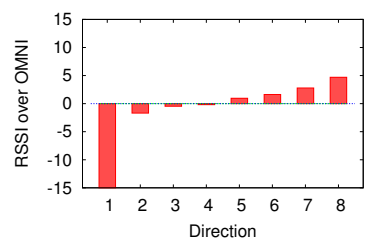

(h) Location h, LOS

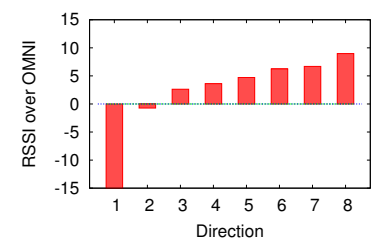

(c) Location c, NLOS

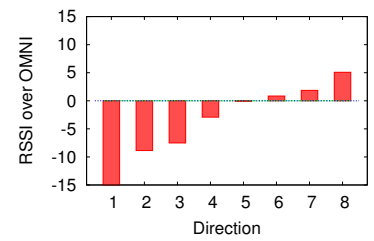

(f) Location f, LOS

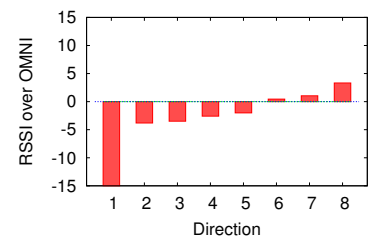

(i) Location i, NLOS

Fig. 9. RSSI over OMNI (in $\mathrm{dB}$ ) at each receiver for pattern set 2 